\title{
Leitlinien-Clearing zur Qualitätssicherung der Gesundheitsversorgung - Leitlinien contra Clearing oder Clearing cum Leitlinien?
}

\author{
Guideline Clearing Promoting the Quality of the Health Care System: \\ Guidelines Contra Clearing or Clearing Cum Guidelines?
}

Im August 1998 einigten sich die Partner der Clearingstelle bei der Ärztlichen Zentralstelle Qualitätssicherung (ÄZQ) über das Verfahren des Leitlinien-Clearings, woraus 1999 das „LeitlinienClearingverfahren“ hervorging. Das übergeordnete Ziel ist es, gemeinsam ein wissenschaftlich begründetes und praktikables Leitlinienprogramm in der Medizin zu fördern und zu unterstützen. Dies sollte in Form eines institutionellen Verfahrens zur „Kritischen Bewertung von Leitlinien“ (so genanntes „LeitlinienClearingverfahren“) umgesetzt werden, das den Kriterien der Transparenz, Praktikabilität, Wissenschaftlichkeit und Wirtschaftlichkeit folgt [1,2]. Einige Unterschiede zwischen Leitlinien auf der einen Seite und dem Leitlinien-Clearing auf der anderen sind in Tab. 1 dargestellt.

Während Leitlinien von Experten der Fachgesellschaften erstellt werden, konstituieren sich die Mitlieder des Expertenkreises für das Leitlinien-Clearingverfahren aus den verschiedenen Versorgungsbereichen des Gesundheitssystems, wie beispielsweise im Falle des Leitlinien-Clearingverfahren „COPD“, aus den Bereichen der Allgemeinmedizin, Rehabilitation, Klinischen Pharmakologie, Epidemiologie, Radiologie und der Inneren Medizin. Unter Moderation der ÄZQ wurden bereits diverse Leitlinien-ClearingVerfahren zum Abschluss gebracht. Hier sei z.B. auf den Bericht zum Asthma bronchiale verwiesen, welcher nach methodischen und inhaltlichen Aspekten getrennt bereits in der Pneumologie als Zusammenfassung veröffentlicht wurde $[3,4]$ und darüber hinaus auch in ausführlicher Form erhältlich ist $[5,6]$.

Im Jahr 2003 haben die Spitzenverbände der Selbstverwaltungskörperschaften im Gesundheitswesen die Durchführung eines Clearingverfahrens zu deutsch- und englischsprachigen Leitlinien zum Thema „COPD“ verabredet, dessen vollständige Fassung
Tab. 1 Charakteristika von Leitlinien und des Leitlinien-Clearings

\begin{tabular}{|c|c|}
\hline Leitlinien & Clearingverfahren \\
\hline $\begin{array}{l}\text { Hilfe für ärztliche Entscheidungs- } \\
\text { prozesse im Rahmen einer leistungs- } \\
\text { fähigen Versorgung }\end{array}$ & Information über Leitlinien \\
\hline $\begin{array}{l}\text { Wesentlicher Bestandteil von } \\
\text { Qualitätssicherung und Qualitäts- } \\
\text { management }\end{array}$ & $\begin{array}{l}\text { Bewertung wichtiger Leitlinien anhand } \\
\text { fest-gelegter Kriterien, ggf. Empfeh- } \\
\text { lungen zur Verbesserung }\end{array}$ \\
\hline $\begin{array}{l}\text { Instrumente aller Versorgungs- } \\
\text { bereiche zur Verbesserung der Er- } \\
\text { gebnisse, zur Minimierung von } \\
\text { Handlungsrisiken und zur Erhöhung } \\
\text { der Wirtschaftlichkeit }\end{array}$ & $\begin{array}{l}\text { Kennzeichnung der für gut befunde- } \\
\text { nen Leitlinien }\end{array}$ \\
\hline $\begin{array}{l}\text { Integratives Mittel zur Vernetzung } \\
\text { der Versorgungsbereiche }\end{array}$ & $\begin{array}{l}\text { Unterstützung bei der Verbreitung } \\
\text { von Leitlinien }\end{array}$ \\
\hline $\begin{array}{l}\text { Hilfe für die ärztliche Aus-, Weiter- } \\
\text { und Fortbildung }\end{array}$ & $\begin{array}{l}\text { Koordination von Erfahrungsberichten } \\
\text { über bewertete Leitlinien }\end{array}$ \\
\hline $\begin{array}{l}\text { Instrument kontinuierlichen Lernens } \\
\text { aus Wissenschaft und Praxis }\end{array}$ & $\begin{array}{l}\text { Unterstützung bei der Evaluation von } \\
\text { Leitlinien }\end{array}$ \\
\hline $\begin{array}{l}\text { Vorzugsweise Experten einer Fach- } \\
\text { gesellschaft }\end{array}$ & $\begin{array}{l}\text { Experten aus den verschiedenen } \\
\text { Versorgungsbereichen }\end{array}$ \\
\hline
\end{tabular}

soeben publiziert wurde $[5,7]$ und dessen Zusammenfassung in dieser Ausgabe der Zeitschrift abgedruckt ist [8]. Wie hierbei deutlich wird, geht es bei dem Leitlinien-Clearing nicht um die Erstellung systematischer Entscheidungshilfen über die angemessene Vorgehensweise bei speziellen gesundheitlichen Problemen. Ziel dieses Verfahrens ist es vielmehr, im Sinne des oben definierten Leitlinien-Clearings eine „Sichtung“ der wesentlichen Elemente einer Leitlinie vorzunehmen. Entsprechend den 
Vorgaben eines Clearingverfahrens [1] handelt es sich bei den im Leitlinien-Clearingbericht „COPD“ getroffenen Aussagen also ausschließlich um die Darstellung methodischer und inhaltlicher Eckpunkte, die gleichsam als ein Gerüst für künftige Leitlinien dienen sollen. Dabei werden systematisch alle potenziell relevanten Aspekte berücksichtigt. Die getroffenen Feststellungen werden detailliert begründet und im Einzelnen mit Beispielen aus den evaluierten Leitlinien illustriert.

Es geht also nicht um ein Leitlinien-Clearing contra Leitlinien, sondern vielmehr um ein Leitlinien-Clearing cum Leitlinien, wobei das Clearingverfahren die relevanten Eckpunkte einer praktischen und auf nachvollziehbaren Inhalten beruhenden Leitlinie hervorhebt. Die konkrete Umsetzung der Empfehlungen für die Praxis sowie die Implementierung obliegt dann den Leitlinienerstellern

\section{Literatur}

${ }^{1}$ Bundesärztekammer, Kassenärztliche Bundesvereinigung. Das Leitlinien-Clearingverfahren von Bundesärztekammer und Kassenärztlicher Bundesvereinigung in Zusammenarbeit mit der Deutschen Krankenhausgesellschaft und den Spitzenverbänden der Gesetzlichen Krankenversicherungen. Ziele und Arbeitsplan. Dtsch Ärztebl 1999; 96: A-2105-2106

2 Ärztliche Zentralstelle Qualitätssicherung. Checkliste „Methodische Qualität von Leitlinien“. Dtsch Arztebl 2000; 97: A1170-1172

${ }^{3}$ Thole H, Weingart O, Lampert U et al. Das Leitlinien-Clearingverfahren Asthma bronchiale - 1. Methodik und Ergebnisse der formalen Bewertung. Pneumologie 2003; 57: 459-467

${ }^{4}$ Thole H, Kroegel C, Bassler D et al. Das Leitlinien-Clearingverfahren Asthma bronchiale - 2. Empfehlungen zu Eckpunkten für eine nationale Leitlinie Asthma bronchiale. Pneumologie 2004; 58: 165 - 175

5 Ärztliches Zentrum für Qualität in der Medizin (äzq). http://www.leitlinien.de/clearingverfahren/index/clearingberichte/index/copd/ 00copd/view

${ }^{6}$ Bassler J, Forster J, Franzen D et al. Leitlinien-Clearingbericht „Asthma bronchiale“, Schriftenreihe der Ärztlichen Zentralstelle Qualitätssicherung, Band 9. Hrsg: Zentralstelle der Deutschen Ärzteschaft zur Qualitätssicherung in der Medizin, 1. Auflage. München, Wien, New York: Zuckschwerdt-Verlag, 2002

${ }^{7}$ Fessler J, Fischer J, Franzen D et al. Leitlinien-Clearingbericht „COPD“, Schriftenreihe der Ärztlichen Zentralstelle Qualitätssicherung, Band 15. Hrsg: Zentralsstelle der Deutschen Ärzteschaft zur Qualitätssicherung in der Medizin. 1. Auflage. Niebüll: Verlag videel OHG, 2003

${ }^{8}$ Franzen D, Fessler J, Fischer J et al. Das Leitlinien-Clearingverfahren COPD. Empfehlungen für eine nationale Leitlinie. Pneumologie 2004; 58: $860-864$ 